\title{
Optimization of Co-Rotating Twin-Screw Extruders Using Pareto Local Search
}

\author{
C. Teixeira*, J.A. Covas*, T. Stützle**, A. Gaspar-Cunha* \\ * IPC/I3N - Institute of Polymers and Composites, University of Minho, Guimarães, \\ Portugal. \\ ** IRIDIA, CoDE, Université Libre de Bruxelles, Brussels, Belgium. \\ E-mail: cteixeira,@dep.uminho.pt, jcovas,@dep.uminho.pt, stuetzle@ulb.ac.be, \\ agc@dep.uminho.pt
}

\begin{abstract}
A Pareto Local Search (PLS) algorithm was developed and applied to the screw configuration of co-rotating twin-screw extruders. This problem can be seen as a sequencing problem where a set of different screw elements are to be sequentially positioned along the screw in order to maximize the extruder performance. The results obtained were compared with previous results obtained with a Multi-Objective Evolutionary Algorithm (MOEA), which was previously developed by the authors. These results show that the PLS algorithm, despite its conceptual simplicity, is able to generate screws with good performance.
\end{abstract}

\section{Introduction}

Due to their geometrical flexibility and good mixing capacity, co-rotating twin screw extruders are widely used in the polymer compounding industry. This type of machines can easily be adapted to work with different polymeric systems, e.g., polymer blends, nanocomposites or highly filled polymers, taking into account its modular construction. However, this geometrical flexibility makes the performance of these machines strongly dependent on the screw configuration being used, i.e., defining the adequate screw geometry to use in a specific polymer system is an important process requirement. This can be seen as an optimization problem involving the selection of the location of a set of available screw elements along the screw axis.

In the case tackled in this paper, the optimization consists in permuting a specific number of different screw elements in order to maximize the global performance of the system. This problem was designated as Twin Screw Configuration Problem (TSCP); it is a sequencing problem with, the aim of determining the posi- 
tion of screw elements along the screw axis. Since it involves several conflicting objectives, it is actually a multi-objective combinatorial optimization problem (MCOP).

The TSCP was previously tackled using a Multi-Objective Evolutionary Algorithm (MOEA) [1]. One important limitation of using MOEAs is the high number of necessary evaluations of the objective functions, since this implies running a numerical modelling routine, which has significant computational costs. Therefore, in the present work a Stochastic Local Search (SLS) algorithm was applied as an alternative for tackling this problem. In particular, we adopted the Pareto Local Search (PLS) strategy [2,3]. For that purpose, a detailed comparison to the previously designed MOEA using different objectives was made. In particular, this work is a part of a more comprehensive study where different approaches will be tested, including MOEA, Multi-Objective Ant Colony Optimization (MOACO), simple SLS algorithms and hybrids of these three classes of algorithms. The main motivation for the study of PLS here is its conceptual simplicity and its known very good performance.

This paper is organized as follows. In section 2, the twin-screw extrusion configuration problem is described. In section 3 , the details of the algorithms used are discussed. Then, in section 4 the results are presented and discussed and we conclude in section 5 .

\section{Twin-Screw Extruders}

Intermeshing co-rotating twin-screw extruders have by two Archimede-type screws with the same geometry rotating in the same direction inside a heated barrel [4]. The screws are usually built up by coupling individual screw elements with different geometries. Conveying, mixing and kneading elements are available, with distinct geometries. The performance of this type of extruders depends on the use of the correct sequence of elements, so that the extruder be able to accomplish its main functions, namely, transporting and melting the solid polymer, mixing and devolatilizing and forcing the polymer to pass trough the die [4]. Polymer pellets or powder are usually fed inside the barrel at a pre-set feed rate. The rotation speed of the screws, together with the local temperatures and screw geometry subject the polymer to a variety of thermomechanical stresses along the screw axis.

Therefore, the co-rotating twin-screw configuration problem consists in defining the best location of a set of screw elements along the screw shaft as illustrated in figure 1. In this example the aim is to determine the position along the screw of 10 transport elements, 3 kneading blocks (with different staggering angles) and one reverse element.

The performance of each screw configuration is obtained by using an elaborated computer simulation of the polymer flow through the screw elements, taking into account the relevant thermal and rheological physical phenomena. The proc- 
ess comprises the following steps: (i) transport of solid material; (ii) melting; (iii) mixing and homogenisation; (iv) pressure generation and (v) flow through the die $[5,6]$. The flow characteristics are determined by the different geometries of the screw elements. Right handed elements have conveying properties while left handed and kneading blocks with a negative staggering angle create a flow restriction (generating pressure).

The computer simulation programme considers all above steps [6]. After the solid polymer is fed into the hopper, it will flow under starved conditions through transport elements. When a restrictive element is reached, the channel starts to fill up and the melting process is initiated. When the polymer is full melted, the flow develops with or without pressure in the remaining of the screw elements, depending on whether they are totally or partially filled; overall, the pressure is determined by the location of the restrictive elements. Each evaluation of a screw configuration takes about one to two minutes on current CPUs. Hence, the high computational effort required for these evaluations is an additional complicating factor and we require algorithms that use a low number of function evaluations.

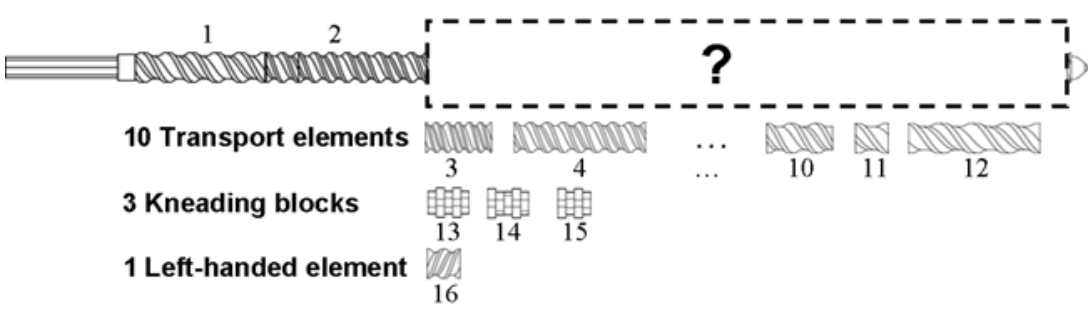

Fig. 1. Screw elements.

\section{Multi-Objective Optimization}

\subsection{Multi-Objective Evolutionary Algorithms}

MOEAs have been recognized in the last decade as good methods to explore and find an approximation to the Pareto-optimal front for multi-objective optimization problems. This is due to the difficulty of traditional exact methods to solve this type of problems and by their capacity to explore and combine various solutions to find the Pareto front in a single run. A MOEA must provide a homogeneous distribution of the population along the Pareto frontier, together with an improvement of the solutions along successive generations [7, 8].

In this work, the Reduced Pareto Set Genetic Algorithm (RPSGA) is adopted $[9,10]$, where a clustering technique is applied to reduce the number of solutions on the efficient frontier. Initially, RPSGA sorts the individuals of the population in 
a number of pre-defined ranks using a clustering technique, in order to reduce the number of solutions on the efficient frontier, while maintaining its characteristics intact. Then, the individuals' fitness is calculated through a ranking function. To incorporate this technique, the traditional GA was modified $[9,10]$, following the steps of a traditional GA, except for the existence of an external (elitist) population and for a specific fitness evaluation. Initially, an internal population of size $N$ is randomly defined and an empty external population formed. At each generation a fixed number of best individuals, obtained by reducing the internal population with the clustering algorithm [10], are copied to an external population. This process is repeated until the number of individuals of the external population becomes full. Then, the RPSGA is applied to sort the individuals of the external population, and a pre-defined number of the best individuals are incorporated in the internal population by replacing low fitness individuals. Detailed information about this algorithm can be found elsewhere [9, 10].

\subsection{Pareto Local Search Algorithm}

SLS algorithms [11] have been successfully applied to single objective problems and, more recently, also to Multi-Objective Optimization Problems (MOOP). Successful single-objective based SLS algorithms can be readily extended to MOOPs via two strategies. The one we study here is to adopt a component-wise acceptance model, where a new solution is accepted in the local search if it is non-dominated by any of the other previous. As an example of such a strategy, we use Pareto Local Search (PLS) [2,3]. A key component of any local search algorithm is the definition of which solutions are neighbouring. Some preliminary experiments showed that the most suitable neighbourhood relation to be used in the local search is based on the 2-swap operator: two solutions are considered to be neighbours, if one can be obtained from the other by swapping the position of two screw elements.

The main ideas of PLS are the use of an archive, where all non-dominated solutions found so far are kept, and the exploration of the neighbourhood of each of these solutions using non-dominance criteria to decide about the acceptance of solutions [2,3]. The algorithm starts with a random initial solution. This is added to the archive and its neighbourhood is explored using the 2-swap operator. All nondominated solutions identified in the neighbourhood exploration are added to the archive, if they are not dominated by any of the solutions in the archive, otherwise it is eliminated. These solution selection and archive update steps are iterated until the neighbourhood of all solutions in the archive has been explored. In order to avoid a too strong increase of the number of solutions in the archive, an archive bounding technique is used [12]. This bounding technique divides the objective space by a grid into hypercubes and allows only one non-dominated solution to occupy a given hypercube. 


\section{Results and Discussion}

\subsection{Case study}

The RPSGA and the PLS algorithms presented above were tested using the individual screw elements presented in Table 1 for a Leistritz LSM 30.34 twin-screw extruder. In this example the objectives considered are the average strain, the specific mechanical energy (SME) and the viscous dissipation. Four instances and three different case studies were considered as presented, respectively, in Tables 1 and 2. Each optimization run was performed 10 times using different seed values. The comparison between the algorithms was made using the attainment functions methodology [13].

Table 1. Configuration of the individual screw elements for the 4 instances considered

\begin{tabular}{|c|c|c|c|c|c|c|c|c|c|c|c|c|c|c|}
\hline Instance & $\begin{array}{l}\text { Screw } \\
\text { Element }\end{array}$ & 1 & 2 & 3 & 4 & 5 & 6 & 7 & 8 & 9 & 10 & 1112 & 13 & 141516 \\
\hline \multirow[b]{2}{*}{ TSCP1 } & Length (mm) & 97.5 & 120 & 45 & 60 & 30 & 30 & 30 & 60 & 30 & 120 & 30120 & 37.5 & $60 \quad 60 \quad 30$ \\
\hline & Pitch (mm) & 45 & 30 & 45 & 30 & 20 & 60 & 30 & 20 & $\begin{array}{l}\text { KB } \\
-60^{\circ}\end{array}$ & 30 & 3060 & 20 & 453020 \\
\hline \multirow[b]{2}{*}{ TSCP2 } & Length (mm) & 97.5 & 120 & 45 & 60 & 30 & 30 & 30 & 60 & 30 & 120 & 30120 & 37.5 & 606030 \\
\hline & Pitch (mm) & 45 & 30 & 45 & 30 & -20 & 60 & 30 & 20 & $\begin{array}{l}\mathrm{KB} \\
-60^{\circ}\end{array}$ & 30 & 3060 & 20 & 453020 \\
\hline \multirow[b]{2}{*}{ TSCP3 } & Length (mm) & 97.5 & 120 & 45 & 60 & 30 & 30 & 30 & 60 & 30 & 120 & 30120 & 37.5 & $60 \quad 6030$ \\
\hline & Pitch (mm) & 45 & 30 & $\begin{array}{l}\text { KB } \\
-45^{\circ}\end{array}$ & 30 & -20 & 60 & 30 & 20 & $\begin{array}{l}\mathrm{KB} \\
-60^{\circ} \\
\end{array}$ & 30 & 3060 & 20 & 453020 \\
\hline \multirow[b]{2}{*}{ TSCP4 } & Length (mm) & 97.5 & 120 & 45 & 60 & 30 & 30 & 30 & 60 & 30 & 120 & 30120 & 37.5 & $60 \quad 6030$ \\
\hline & Pitch (mm) & 45 & 30 & $\begin{array}{l}\mathrm{KB} \\
-45^{\circ}\end{array}$ & 30 & -20 & 60 & 30 & 20 & $\begin{array}{l}\mathrm{KB} \\
-60^{\circ}\end{array}$ & 30 & 3060 & $\begin{array}{l}\mathrm{KB} \\
-30^{\circ}\end{array}$ & 453020 \\
\hline
\end{tabular}

Table 2. Optimization objectives, aim of optimization and prescribed range of variation used in each case

\begin{tabular}{lllll}
\hline & Objectives & Aim & $\mathrm{X}_{\min }$ & $\mathrm{X}_{\max }$ \\
\hline Case & Average Strain & Maximize & 1000 & 15000 \\
study 1 & Specific mechanical energy & Minimize & 0.1 & 2 \\
Case & Average Strain & Maximize & 1000 & 15000 \\
study 2 & Viscous dissipation & Minimize & 0.9 & 1.5 \\
Case & Specific mechanical energy & Minimize & 0.1 & 2 \\
study 3 & Viscous dissipation & Minimize & 0.9 & 1.5 \\
\hline
\end{tabular}




\subsection{Comparative Results}

In order to demonstrate the capacity of the PLS algorithm to deal with the TSCP, figure 2 shows a Pareto front for case study two and instance 1, considering a single run. As expected, the viscous dissipation (to be minimized) increases with the average strain (to be maximized). The viscous dissipation (measured as the ratio between the average melt temperature and the set barrel temperature) is smaller when the restrictive elements are separated by conveying elements, since in this case the increase in temperature is also smaller. The opposite is true for the case of the average strain.

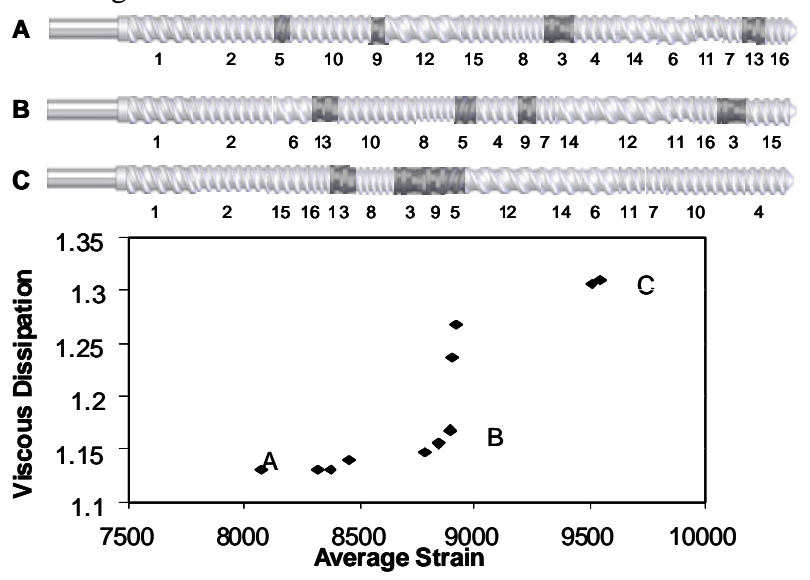

Fig. 2. Pareto front for case study 2, instance 1 and run 1 and some screw configurations generated.

The results obtained with the PLS algorithm presented here were compared with the results obtained with the RPSGA algorithm developed previously. The comparison was made using the EAFs, as shown in Figure 3 for all cases studies and for instance 4. Similar results can be found on www.dep.uminho.pt/agc/results. The graphs on the left represent the region(s) of the Pareto front where the MOEA (in this case the RPSGA) is better, while the graphs on the right represent the region(s) where the PLS algorithm is better. The results shown in Figure 3 allow one to conclude that the PLS is better for instance 4. The same happens for instance 3, while for instance 1 and 2 the results are slightly better for the RPSGA algorithm.

\section{Conclusions}

A Pareto Local Search algorithm was applied with success to the Twin Screw Configuration Problem. The solutions obtained comply with the available scien- 
tific and technical knowledge on the process. The good performance obtained with the PLS algorithm alone is somewhat surprising, since it is a very simple method. In addition, the results indicate that it may be worthwhile to further develop the method, for example, by a better choice of which solutions are to be explored next, or to consider it for a possible combination with other methods, for example, as a post-processor to improve the search process of techniques such as the RPSGA.

\section{References}

1. A. Gaspar-Cunha, J.A. Covas and B.Vergnes. Defining the Configuration of Co-Rotating Twin-Screw Extruders With Multiobjective Evolutionary Algorithms. Polymer Engineering and Science, 1159-1173.

2. L. Paquete, T. Stützle, A study of stochastic local search algorithms for the biobjective QAP with correlated flow matrices, European J. of Operational Research, 169:943-959, 2006.

3. L. Paquete, M. Chiarandini, and T. Stützle. Pareto local optimum sets in the biobjective traveling salesman problem: An experimental study. In X. Gandibleux, M. Sevaux, K. Sörensen and V. T'kindt, editors, Metaheuristics for Multiobjective Optimisation, volume 535 in Lecture Notes in Computer Science, 177-200, Springer Verlag, Berlin, 2004.

4. J.L. White, Twin Screw Extrusion; Technology and Principles, Hanser, Munich, 1990.

5. B. Vergnes, G. Della Valle, L. Delamare, A Global Computer Software for Polymer Flows in Corotating Twin Screw Extruders, Polymer Engineering and Science, 38, p. 1781-1792, 1998.

6. C. Teixeira, A. Gaspar-Cunha, J.A. Covas, A Global Plasticating Software for Co-Rotating Twin Screw Extruders, Under preparation, 2009.

7. A. Gaspar-Cunha, P. Oliveira, J.A. Covas, Use of Genetic Algorithms in Multicriteria Optimization to Solve Industrial Problems, Seventh Int. Conf. on Genetic Algorithms, Michigan, USA, 1997.

8. K. Deb, Multi-Objective Optimization using Evolutionary Algorithms, Wiley, 2001.

9. A. Gaspar-Cunha, J.A. Covas, J.A. - RPSGAe - A Multiobjective Genetic Algorithm with Elitism: Application to Polymer Extrusion. In X. Gandibleux, M. Sevaux, K. Sörensen and V. T'kindt, editors, Metaheuristics for Multiobjective Optimisation, volume 535 in Lecture Notes in Computer Science, 221-249, Springer Verlag, Berlin, 2004.

10. A. Gaspar-Cunha, Modelling and Optimization of Single Screw Extrusion, PhD Thesis, University of Minho, Guimarães, Portugal, 2000 (Downloadable from website http://www.lania.mx/ ccoello/EMOO/).

11. Holger H. Hoos and Thomas Stützle. Stochastic Local Search - Foundations and Applications. Morgan Kaufmann Publishers, San Francisco, California, USA, 2004.

12. E. Angel, E. Bampis and L. Gourvés. A dynasearch neighborhood for the bicriteria traveling salesman problem. In X. Gandibleux, M. Sevaux, K. Sörensen and V. T'kindt, editors, Metaheuristics for Multiobjective Optimisation, volume 535 in Lecture Notes in Computer Science, 153-176, Springer Verlag, Berlin, 2004.

13. V. G. da Fonseca, C. Fonseca and A. Hall. Inferential performance assessment of stochastic optimisers and the attainment function. In E. Ziztler, K. Deb, L. Thiele, C.A. Coello Coello, and D. Corne, editors, Evolutionary Multi-Criterion Optimization (EMO 2001), Lectures Notes in Computer Science 1993, 213-225. Springer Verlag, 2001. 

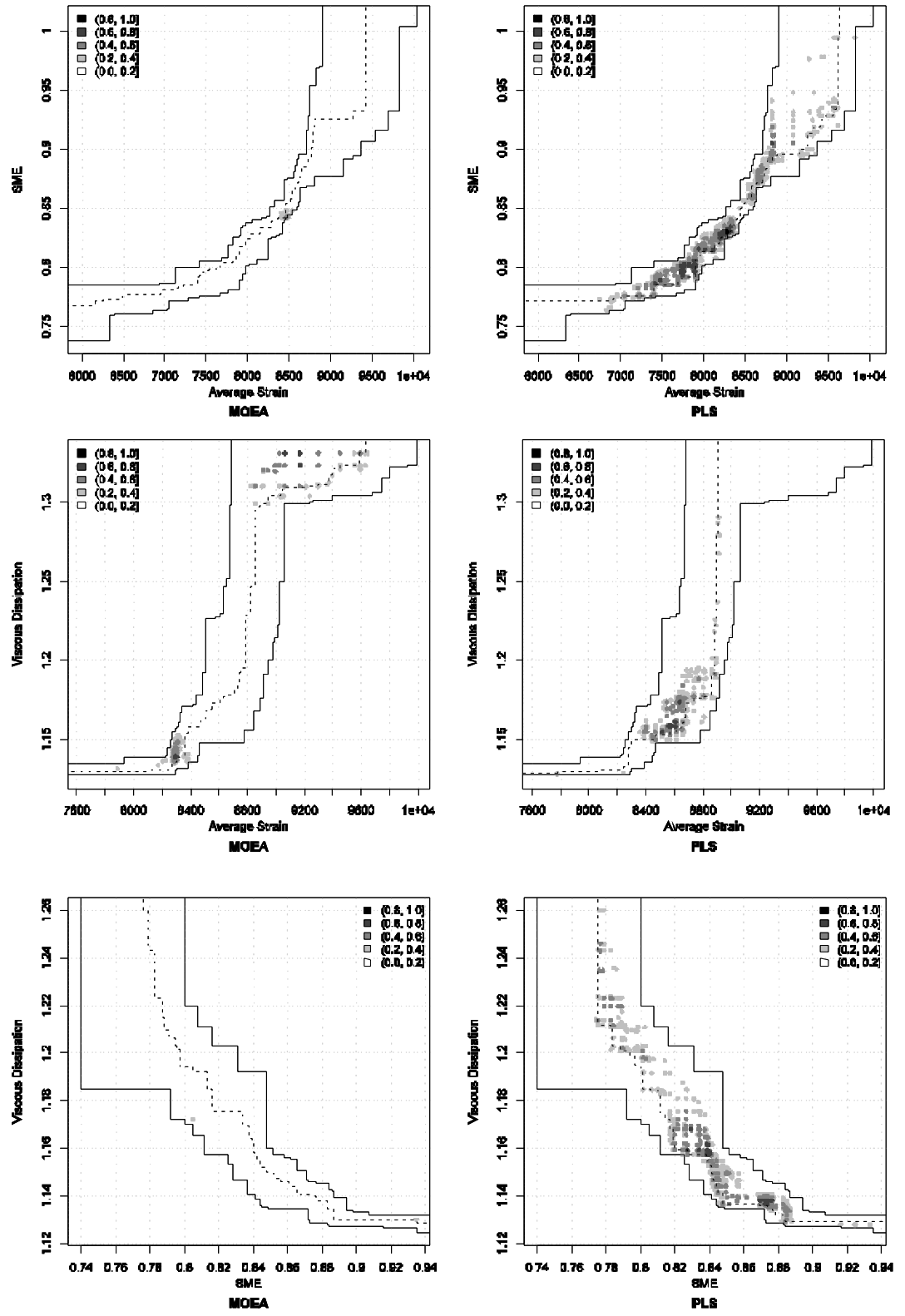

Fig. 3. EAFs differences between MOEA and PLS results for instance 4 and case studies 1 (top), 2 (middle) and 3 (bottom). 